\title{
Aspectos técnicos de la fijación percutánea de las fracturas de escafoides
}

\author{
C. Heras Palou \\ CONSULTANT HAND SURGEON \\ Correspondencia: \\ Clinical Director Pulvertaft Hand Centre \\ London Road \\ Derby DE1 2QY \\ England \\ Tel 00441332347141 ext 2396 \\ E-mail: carlos.heras-palou@virgin.net
}

La fijación percutánea de fracturas del escafoides se ha establecido como una opción quirúrgica en los últimos años. Esta es una técnica fiable y efectiva, pero con poco margen de error. Se describen los aspectos técnicos para hacer este procedimiento más fácil y evitar complicaciones.

Palabras clave: Osteosíntesis Percutánea, Escafoides, Técnica, Fractura.
Perccutaneous fixation of fractures of the scaphoid has been established as a surgical option in the past few years. It is a reliable and effective technique but the margin for error is small. Technical aspects to make this procedure easier, and to avoid complications, are described.

Key words: Osteosintesis Percutánea, Escafoides, Técnica, Fractura.

Rev. Iberam. Cir. Mano - Vol. 34 - Núm. 2 - noviembre 2006 (27-32)

\section{INTRODUCCIÓN}

$\checkmark$ 1 concepto de la fijación percutánea de fracturas del escafoides no es nuevo ${ }^{1}$. En los años 90 sucedieron dos avances tecnológicos que han hecho a la técnica popular El primero fue la mejora en los aparatos de fluoroscopia disponibles, y el segundo la aparición en el mercado de tornillos canulados para la osteosintesis del escafoides. Esto ha hecho que la fijación percutánea se convierta en una técnica relativamente fácil, con resultados clínicos excelentes y con un análisis de coste económico favorable.

\section{TECNICA}

En la literatura anglosajona la fijación percutánea del escafoides ha sido impulsada por el Dr Slade ${ }^{2}$ de EEUU, de forma anterograda (des- de el dorso de la muñeca, de proximal a distal), y por el Dr. Goddard ${ }^{3}$ de Inglaterra usando la forma retrógrada (por vía palmar de distal a proximal).

La cirugía se realiza con el paciente en decúbito supino y con anestesia regional según se requiera. La fijación anterograda exige la flexión de la muñeca afectada y la introducción de una aguja de Kirschner desde el dorso en dirección hacia la base del pulgar. El punto de entrada en el escafoides es en el polo proximal, en la vecindad de la inserción del ligamento escafo-semilunar, y la dirección debe ser el eje longitudinal del escafoides. Con la muñeca en flexión el escafoides aparece como un cilindro cuando su eje longitudinal es co-linear con los rayos $\mathrm{X}$, y el punto de inserción debe ser en el centro del círculo.

Esta técnica se puede realizar completamente percutánea o a través de un incisión peque- 


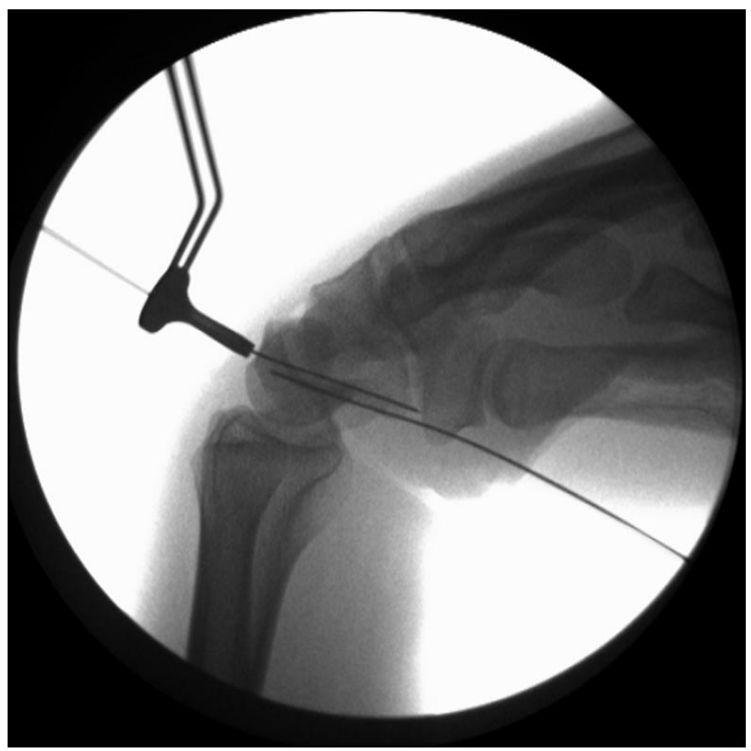

Figura 1: En la técnica anterograda la aguja de Kirshner se introduce de proximal a distal.

ña. Nosotros preferimos una incisión de $2 \mathrm{~cm}$. sobre el tubérculo de Lister, que nos permite ver los tendones extensores y protegerlos y también identificar exactamente el punto de entrada de la aguja en el hueso.

En principio la fijación anterograda percutánea parece ideal para fracturas no desplazadas del escafoides proximal. Es importante que la posición de la aguja, y en consecuencia del tornillo, sea central en el escafoides y esto es mas fácil de conseguir con la técnica anterograda que con la retrograda debido a la geometría del escafoides.

Las desventajas de la fijación anterograda son las siguientes:

- Es más difícil orientarse, ya que no se pueden conseguir las proyecciones radiográficas habituales, a no ser que uno avance las agujas, sacándolas por el lado palmar. En esta maniobra se corre el riesgo de perder presa y de dañar estructuras. La verificación final con proyecciones convencionales es posible sólo al final de la intervención (Figuras 1 y 2).

- Al avanzar las agujas hacia distal y palmar, a veces estas pasan a través del trapecio, con el riesgo de dañar la articulación escafotrapezio.

- Esta técnica exige flexión de la muñeca. En general las fracturas del escafoides tienden

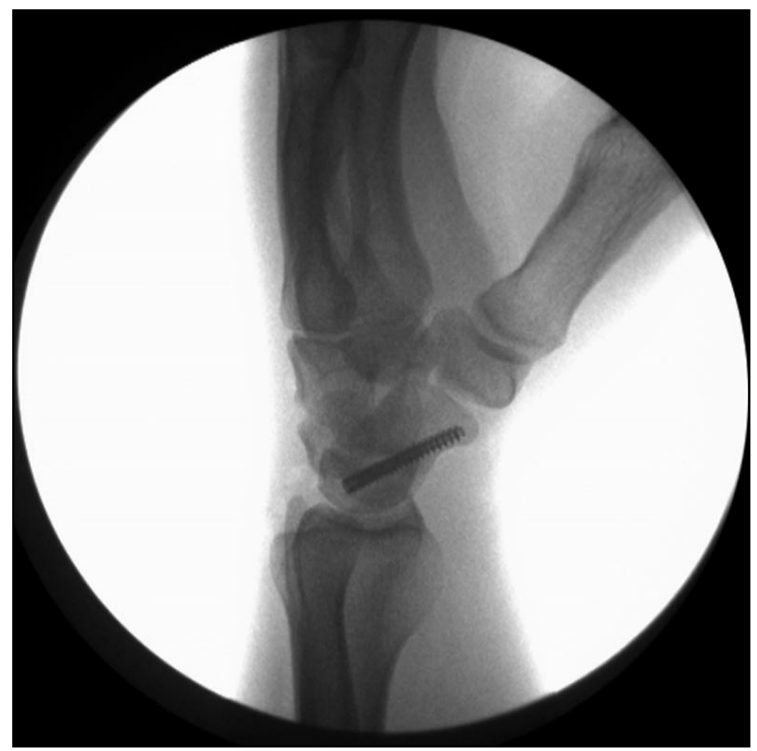

Figura 2: Fijación anterograda con tormillo canulado.

a desplazar en flexión y pronacion, y por ello la posición de la muñeca en flexión y pronacion tiende a aumentar el desplazamiento de la fractura. Esto limita la indicación de esta técnica a casos de fracturas no desplazadas y estables.

- En las fracturas de polo proximal del escafoides, es frecuente que el fragmento proximal es pequeño y por lo tanto un tornillo canulado es demasiado grande y presenta le riesgo de dañar el fragmento que queremos fijar. En estos casos nosotros preferimos visualización del fragmento y la fractura, y fijación con un tornillo pequeño no canulado (1,7 ó 2,3 mm).

Por estas razones, en nuestra institución la técnica anterograda se usa cada vez menos y preferimos la técnica retrograda (palmar de distal a proximal), como describimos a continuación.

Con el paciente en decúbito supino, la muñeca se coloca en supinación y extensión, lo cual tiende a reducir el desplazamiento de la fractura. Se coloca una aguja sobre la piel para marcar la dirección del escafoides con la ayuda de la fluoroscopia (Figura 3). A continuación nosotros usamos la parte metálica de una cánula venosa (calibre14 para una aguja de 1 mm de diámetro) como guía y para proteger las partes blandas (Figura 4a y 4b). Es muy im- 

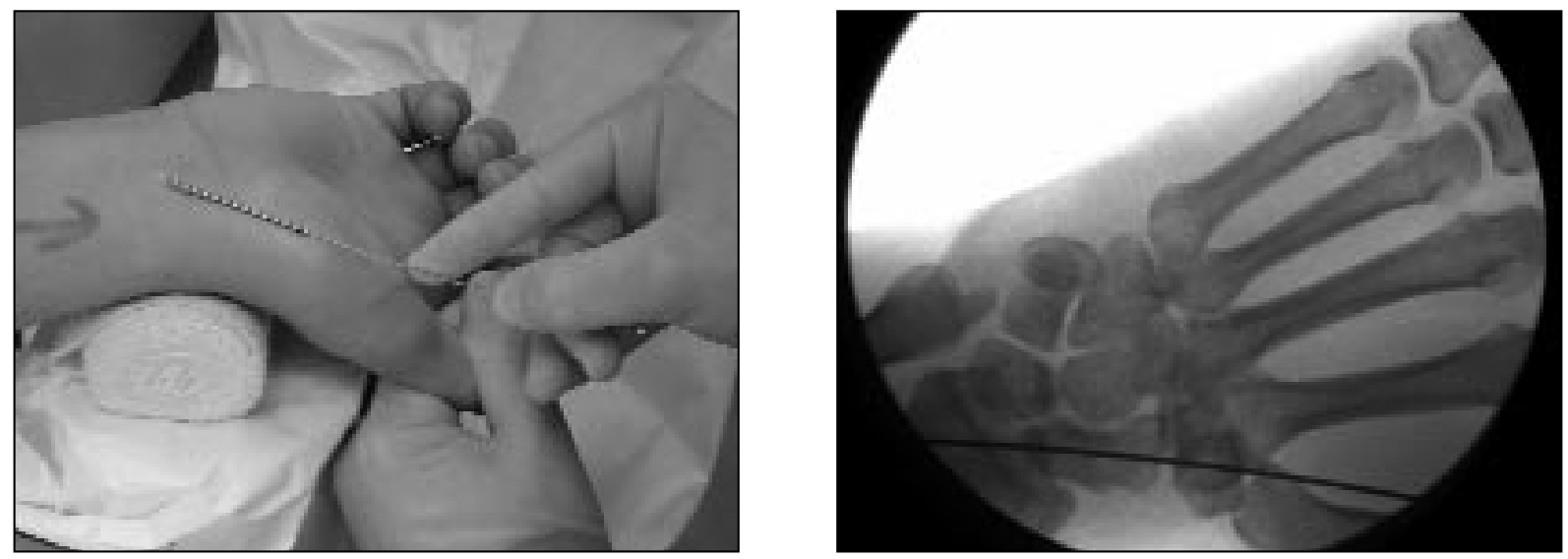

Figura 3 (a y b): Es aconsejable marcar la línea del escafoides. Esto facilita la inserción de la cánula en el punto y con la dirección requerida.

portante que el punto de entrada sea central en el escafoides en la proyección fluoroscópica lateral, ya que la tendencia del punto de entrada es a ser demasiado palmar y luego al fresar se va a dañar la parte palmar del polo distal, y como consecuencia el tornillo no consigue una buena presa en ese fragmento (Figura 5).

Si la fractura es conminuta o inestable, una segunda aguja ayuda a estabilizar los fragmentos durante el fresado y la introducción del tornillo y evita el riesgo de rotación de los fragmentos (Figura 6a y 6b).

Al verificar la posición de la aguja con el fluoroscopio, es importante que la aguja sea perpendicular a los rayos $\mathrm{X}$ (es decir aguja horizontal) y obtener por lo menos dos proyecciones, perpendiculares entre si. Es más fácil pensar en obtener dos proyecciones de la aguja y ver la posición del escafoides alrededor de la aguja, que conseguir las proyecciones habituales del escafoides e intentar entender la posi-

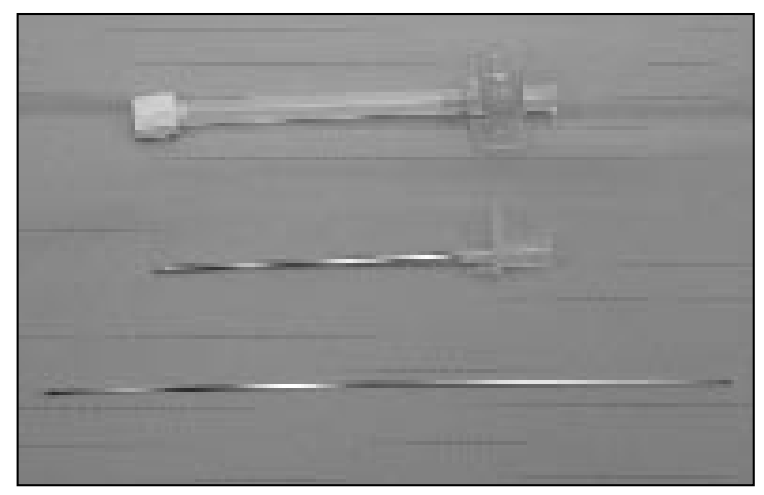

Figura 4: La parte metálica de una cánula venosa funciona muy bien como guía para la inserción de la aguja. ción de la aguja, ya que proyecciones oblicuas de la aguja son difíciles interpretar.

Una vez la posición de la aguja es satisfactoria, se hace un incisión de unos $4 \mathrm{~mm}$ donde la aguja penetra la piel, para permitir el fresado sin dañar la piel.

El fresado se puede hacer a mano o con el uso de instrumental eléctrico o de aire comprimido, pero siempre a bajas revoluciones y a poder ser sin perforar el polo proximal del escafoides. Si la fresa no avanza como se espera, es importante usar la fluoroscopia para confirmar la posición de la fresa y la aguja. Existe el riesgo de que si durante esta maniobra la aguja se dobla, aunque sea ligeramente, la fresa puede dañar y romper la aguja, creando una situación donde un trozo de la aguja rota esta dentro del escafoides, justo en la posición donde desearíamos poner el tornillo. Si el extremo de la aguja protruye en el polo proximal, es obligado retirarla y esto requiere un abordaje dorsal del escafoides. Esta situación es fácil de prevenir, pero difícil de solventar.

Si al retirar la fresa la aguja sale, lo más fácil es introducir la cánula venosa para encontrar el canal fresado y luego introducir otra aguja a través de la cánula.

El error mas común es el de introducir un tornillo demasiado largo. Nosotros recomendamos medir la longitud necesaria y sustraer por lo menos $4 \mathrm{~mm}$. a la hora de elegir tornillo. Cualquiera de los tornillos canulados para la fijación de escafoides disponibles en el mercado en estos momentos produce buenos resultados clínicos siempre que la indicación sea correc- 


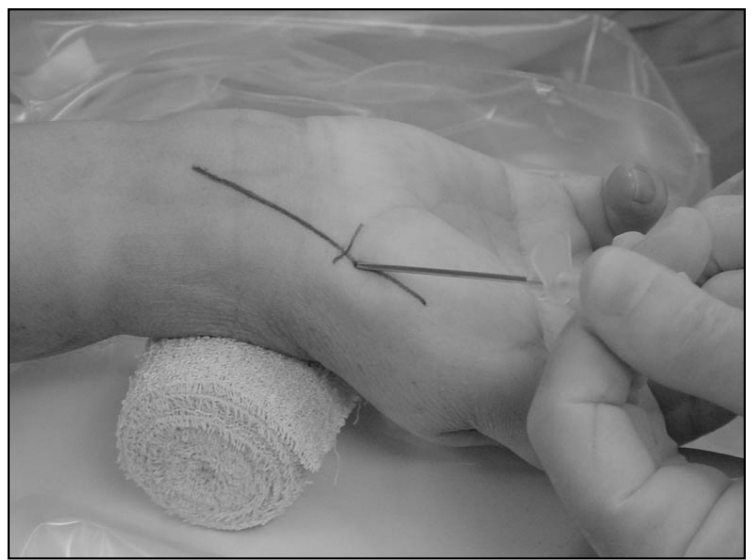

Figura 5 ( a y b): La inserción de la cánula determinara el punto de entrada y la dirección de la aguja. Es importante evitar que le punto de entrada sea demasiado palmar.

ta, la posición del tornillo sea central en el escafoides, se consiga compresión de los fragmentos y una buena fijación.

Normalmente la incisión no necesita sutura (Figura 7a ,b) En la mayoría de pacientes no es necesario aplicar yeso, sino tan solo un vendaje durante la primera semana. Es necesario proteger la fijación y evitar trabajo manual y deportes de contacto durante un periodo de 4 a

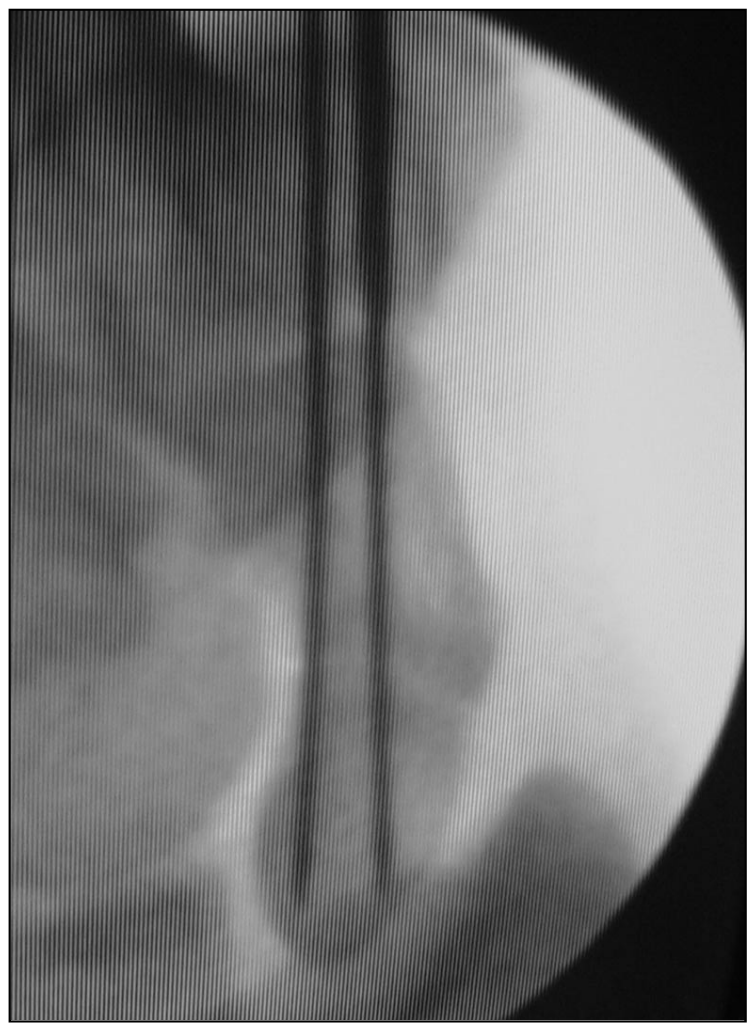

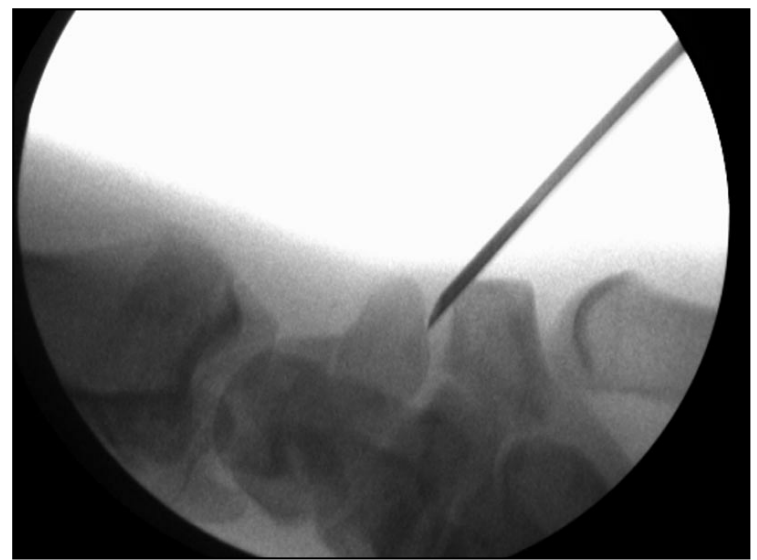

6 semanas, dependiendo del tipo de fractura y actividad del paciente.

Las complicaciones de esta técnica son raras pero existen. Nosotros hemos visto casos de pseduoartrosis y sabemos de un caso de infección con graves consecuencias para el paciente. La causa más común de complicaciones son errores técnicos: tornillo demasiado largo (Figura 8) y tornillo en mala posición (Figura 9a, b), lo cual requiere re-operación sin demora. De lo contrario, la zona expuesta del tornillo dañará el cartílago articular del radio.

Problemas con el instrumental incluyen rotura de la aguja durante el fresado y rotura de la punta del destornillador, lo que puede suceder si el alineamiento del destornillador con el tornillo no es correcto, y puede ser desconcertante ya que súbitamente el tornillo deja de

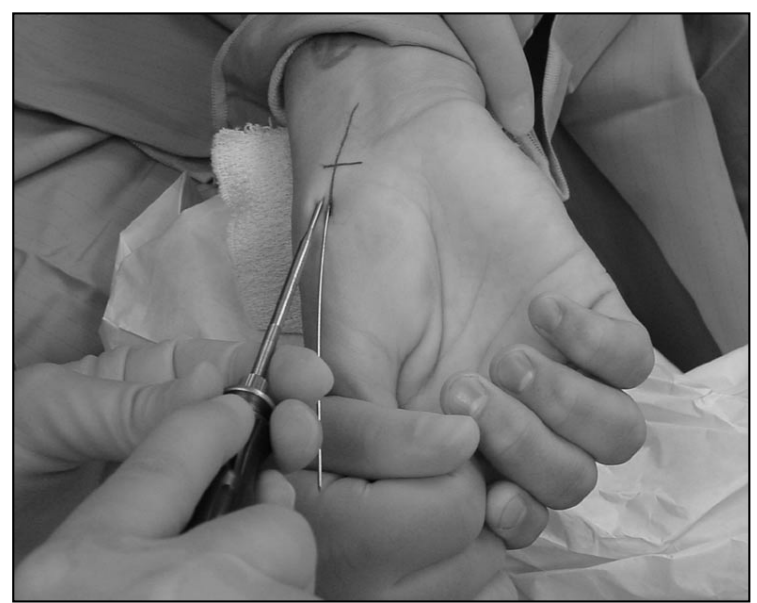

Figura 6 ( a y b): Si la fractura es inestable, una segunda aguja estabiliza la fractura durante el fresado y la introducción del tornillo. 

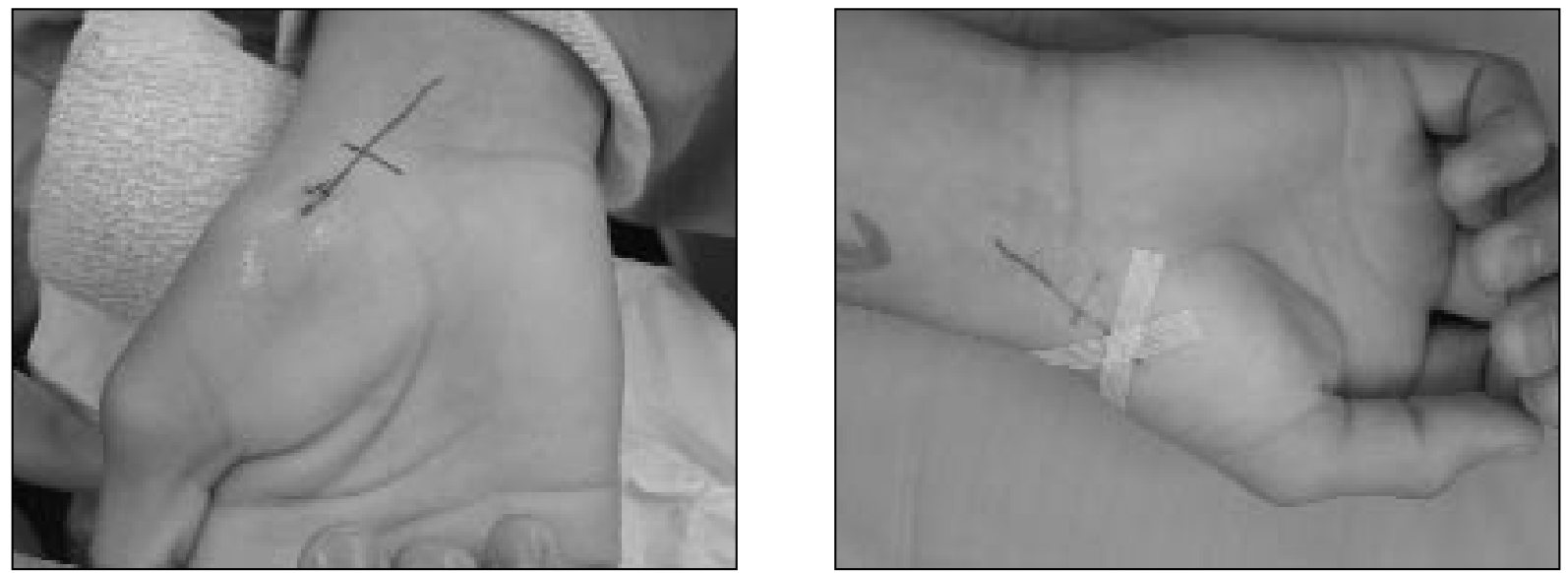

Figura 7 (a y b): La incisión necesaria es de unos 4 mm., y no necesita sutura.

avanzar, y a pesar de girar el destornillador el tornillo ni avanza ni retrocede, sin una causa obvia.

Dolor en lugar de la incisión es infrecuente en nuestra experiencia.

\section{DISCUSIÓN}

La fijación percutaea de las fracturas del escafoides es una técnica fiable y efectiva.

Los análisis de coste económico de la fijación de fracturas del escafoides son favorables ${ }^{4}$,

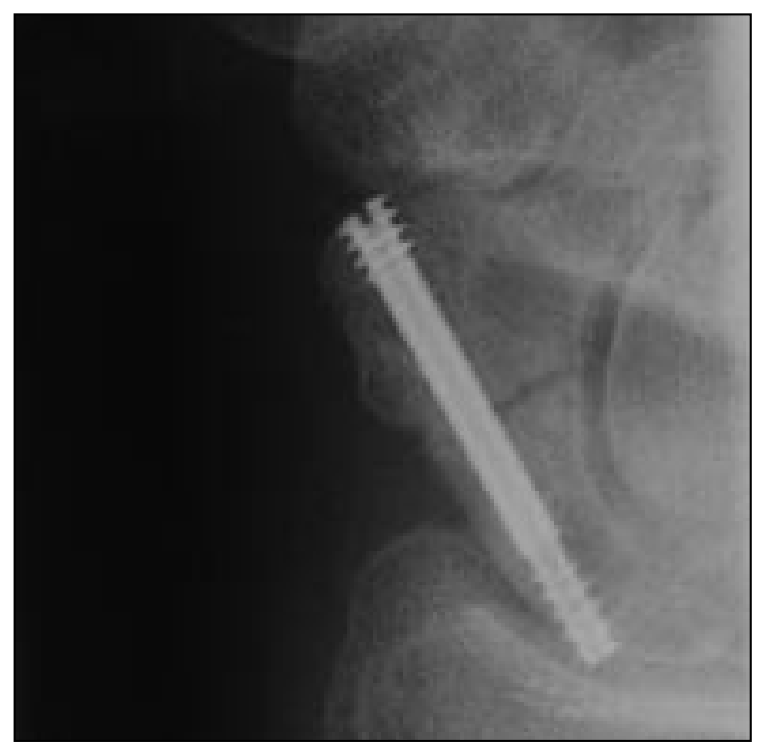

Figura 8: El error técnico más frecuente es poner un tormillo demasiado largo. cuando se comparan al tratamiento ortopédico con yeso. Sin embargo, es importante resaltar que en las series analizadas no había complicaciones significativas, y si hubiera tan solo un caso con una complicación que necesita re-operación, el balance cambia y el tratamiento ortopedia es mejor desde el punto de vista económico y social.

Las indicaciones para la fijación percutánea no están todavía bien delimitadas. En nuestra opinión es importante definir con precisión el tipo de fractura de que se trate. El primer problema es que las radiografías simples no son suficientes para decidir si una fractura del tercio medio del escafoides está desplazada o no. Se necesita una tomografía axial computarizada para poder medir los parámetros necesarios, y es por ello que esta investigación se usa cada vez más. Las fracturas no desplazadas (según el TAC) son de muy buen pronostico y probablemente solo necesitan un periodo de inmovilización mas corto que el habitual de unas 6 a 8 semanas, mientras que las fracturas desplazadas (según el TAC) son una minoría pero con peor pronostico, por lo que son candidatas a osteosintesis percutánea. Este planteamiento evita la intervención no necesaria en la mayoría de las fracturas del escafoides, y ofrece intervención sin demora en la minoría que lo nece$\operatorname{sitan}^{5}$.

Otras indicaciones para la osteosintesis incluyen el requerimiento por las circunstancias del paciente (deportistas profesionales, etc.), 

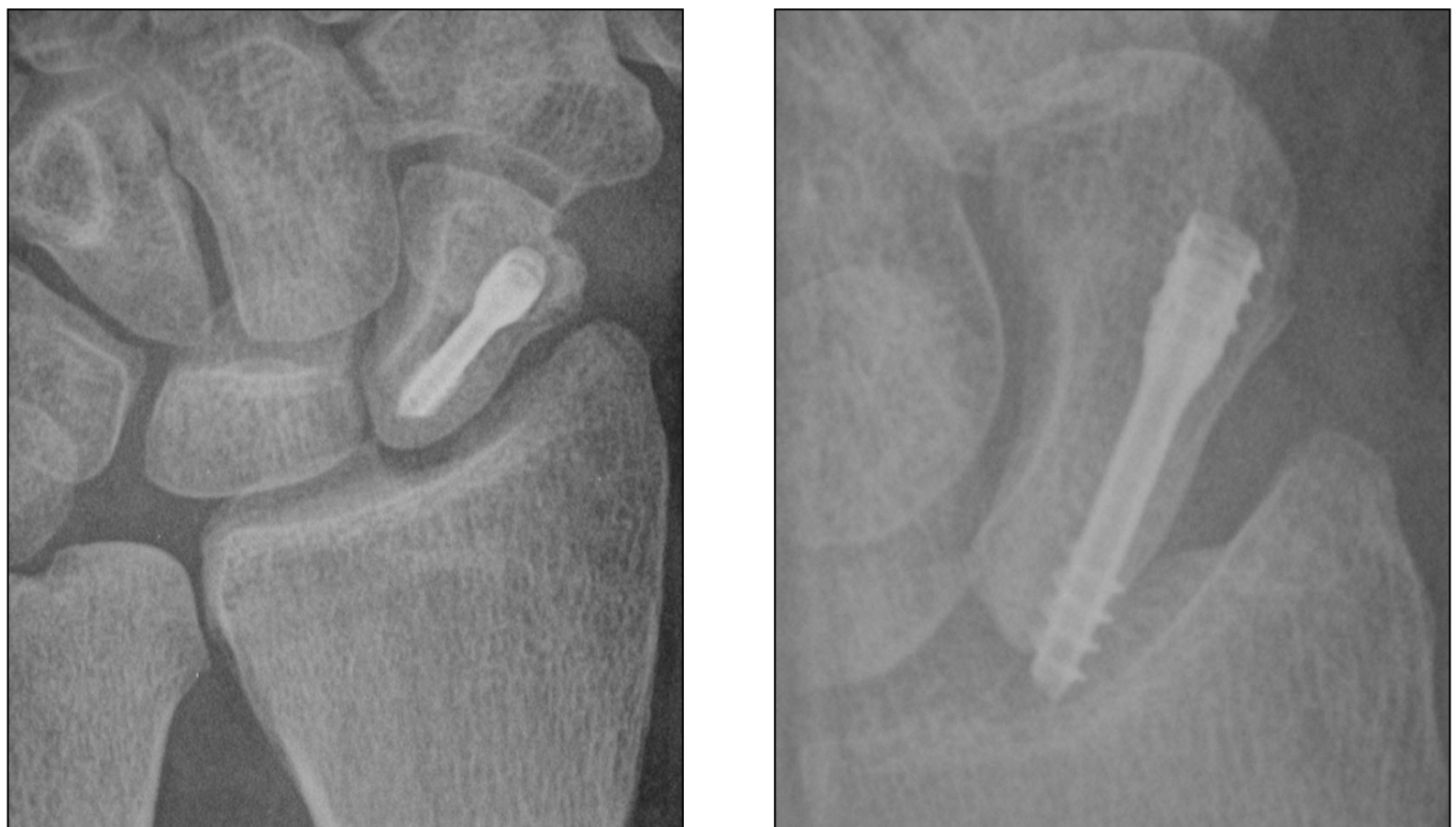

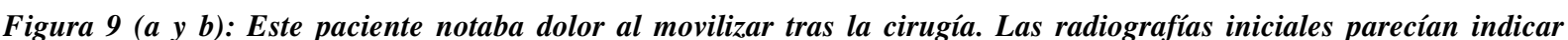
una posición aceptable del tornillo, pero al obtener proyecciones adecuadas del escafoides, es evidente que la posición del tornillo no es aceptable. En la figura 9 a, se puede ver la lesión en la superficie articular del radio causada por el tornillo.

fracturas que se presentan en la consulta más de cuatro semanas después del accidente, y fracturas del escafoides en combinación con otras fracturas o lesiones de la mano o la muñeca.
La fijación percutánea de las fracturas del escafoides es una técnica fiable y efectiva que debemos poner a disposición de nuestros pacientes. El margen de error es mínimo.

\section{BIBLIOGRAFÍA}

1. Wozasek GE, Moser KD. Percutaneous screw fixation for fractures of the scaphoid. JBJS Br 1991; 73: 138-142.

2. Slade JF, Jaskwich D. Percutaneous fixation of scaphoid fractures. Hand Clinics 2001; 17: 553-574.

3. Haddad FS, Goddard NJ. Acu- te percutaneous scaphoid fixation: A pilot study. J Bone Joint Surg Br 1998; 80B: 95-99.

4. Davis EN, Chung KC, Kotsis SV, Lau FH, Vijan S. A cost/utility analysis of open internal reduccion and internal fixation versus case immobilization for acute non-displaced mid-waist scaphoid fractures. Plast Reconstr Surg. 2006 Apr; 117(4) 1223-35.

5. Dias JJ, Wildin CJ, Bhowal B, Thompson JR. Should acute scaphoid fractures be fixed? A randomized controlled trial. J Bone Joint Surg Am. 2005 Oct;87(10): 2160-8. 